\title{
Cumplimiento del tratamiento antibiótico en niños en Atención Primaria
}

\author{
María Rosa Ballester Vidala, Laura de la Rosa Alarcóna ${ }^{a}$ Josefina Mansilla Ortuñob, \\ Gema Tello Nieves ${ }^{c}$, Mercedes de la Ossa Moreno ${ }^{d}$ e Ignacio Párraga Martíneza ${ }^{a}$.
}

\begin{abstract}
a Centro de Salud de La Roda. Albacete. Servicio de Salud de Castilla-La Mancha (España). ${ }^{\text {b }}$ Centro de Salud de Hellín 2. Albacete. Servicio de Salud de Castilla-La Mancha (España). c Gerencia de Atención Integrada de Albacete. Servicio de Salud de Castilla-La Mancha (España).

${ }^{d}$ Gerencia de Atención Integrada de Cuenca. Servicio de Salud de Castilla-La Mancha (España).
\end{abstract}

Correspondencia: María Rosa Ballester Vidal.

Centro de Salud de La Roda. Gerencia de Atención Integrada de Albacete. Servicio de Salud de Castilla-La Mancha.

C/ Mártires, 63.

C.P. 02630. La Roda. Albacete (España).

Correo electrónico:

mrballester@sescam.jccm.es

Este trabajo ha sido presentado en las Primeras Jornadas de Investigación en Atención Primaria de CastillaLa Mancha (Ciudad Real, 21 y 22 de junio de 2013).

Recibido el 16 de enero de 2014.

Aceptado para su publicación el 1 de febrero de 2014.

\section{RESUMEN}

Objetivo: Conocer el cumplimiento terapéutico en niños a los que se prescribe tratamiento antibiótico e identificar los factores relacionados con el incumplimiento.

Diseño del estudio: Estudio descriptivo observacional.

Emplazamiento: Consultas de Pediatría de Atención Primaria.

Participantes: Se evaluaron al cabo de una semana a 117 niños que recibieron tratamiento antibiótico en dos Zonas Básicas de Salud.

Mediciones principales: Se estudiaron variables sociodemográficas (edad, sexo, nacionalidad del niño y padres, nivel de estudios de los padres, estado civil de los padres, clase social de los padres), problemas de salud (clasificación CIAP-2), cumplimiento (cuestionario de MoriskyGreen) y otras características relacionadas con el antibiótico prescrito y con los participantes.

Resultados: La edad media fue de 4,8 años (DE: 3,3). El cumplimiento (Morisky-Green) no fue adecuado en el 55,6\% (IC95\%: 45,1-65,0\%) de los pacientes. Los motivos más frecuentes para la administración incorrecta o abandono del tratamiento fueron: no despertar al niño $(43,8 \%)$, mejoría $(28,1 \%)$ y olvido de toma $(13,8 \%) .14$ pacientes $(12,0 \%)$ presentaron algún efecto adverso. Las variables asociadas, mediante regresión logística, a incumplimiento del tratamiento antibiótico fueron: tener un padre con estudios secundarios o superiores (OR: 3,1; $p=0,020)$, madre con estudios primarios o inferiores $(O R: 3,3 ; p=0,010)$ y realizar 3 tomas de antibiótico diarias (OR: 5,$2 ; \mathrm{p}=0,012$ ), quedando al borde de la significación haber asistido a consulta de pediatría en 2 o más ocasiones en el último mes (OR: 2,2; $\mathrm{p}=0,059$ ).

Conclusiones: Más de la mitad de los niños que toman antibióticos en Atención Primaria no cumple correctamente con el tratamiento prescrito. El nivel de estudios de los padres y el número de tomas al día se relacionan con el incumplimiento del tratamiento antibiótico, lo que se debería considerar al prescribir estos fármacos.

PALABRAS CLAVE: Cumplimiento de la Medicación. Antibióticos. Atención Primaria de Salud.

\section{ABSTRACT}

Antibiotic treatment compliance of children in Primary Care settings.

Object: Collecting information about treatment compliance of children who have been prescribed antibiotics and identifying factors related to treatment noncompliance.

Design of the study: Observational descriptive study.

Setting: Primary Care Pediatric Outpatient Clinics.

Participants: A group of 117 children under antibiotic treatment were assessed one week after prescription in two Basic Healthcare areas.

Main measures: Several socio-demographic variables were studied: age, sex, child and parents' nationality, parents' educational level, marital status of parents and their social status. Health problems (ICPC-2 classification), treatment compliance (Morisky-Green questionnaire) and other characteristics in relation to the prescribed antibiotic and to the participants were also assessed.

Results: The average age of participants was 4.8 years (SD: 3.3$)$. Treatment compliance (Morisky-Green) was inadequate in $55.6 \%$ (CI95\%: $45.1-65.0 \%$ ) of patients.

The most frequent reasons for incorrect administration or treatment discontinuation were: not waking up the child $(43.8 \%)$, improved condition (28.1\%) and forgetting administration (13.8\%). Fourteen patients (12.0\%) presented adverse effects. The associated variables, by logistic regression, to antibiotic treatment noncompliance were: having one of the parents with secondary or higher studies (OR: $3.1 ; \mathrm{p}=0.020)$, mother with primary education or lower (OR: 3.3; $p=0.010$ ) and antibiotic administration in three daily doses (OR:5.2; $p=0.012$ ). The variable "two or more visits to the pediatric clinic in the last month" bordered on statistical significance (OR: 2.2; $p=0.059$ ).

Conclusions: More than half of the children with antibiotic treatment in Primary Care do not comply correctly with the prescribed therapy. The parents' level of studies and the number of daily doses are related to noncompliance with antibiotic treatment, factors which should be considered when those medicines are prescribed.

KEYWORDS: Medication Adherence. Anti-Bacterial Agents. Primary Health Care. 


\section{INTRODUCCIÓN}

El cumplimiento terapéutico es un concepto utilizado en múltiples estudios y, aunque no existe consenso sobre el mismo, la Organización Mundial de la Salud en 2003 se refiere a la adherencia terapéutica como: "el grado en que la conducta de un paciente, en relación con la toma de medicación, el seguimiento de una dieta o la modificación de los hábitos de vida, se corresponde con las recomendaciones acordadas con el profesional sanitario"1. Esta definición está basada en la ya acuñada por Haynes en 1976 sobre el cumplimiento terapéutico ${ }^{2}$; posteriormente se intenta ser menos paternalista; de ahí el término de adherencia o alianza terapéutica de Holmes en $1996{ }^{3}$.

Se ha estudiado el cumplimiento en diferentes patologías crónicas y grupos terapéuticos, aunque con menor frecuencia se han realizado estudios sobre procesos agudos como las patologías infeccio$\mathrm{sas}^{4}$. La repercusión de una adherencia inadecuada a los antibióticos afecta tanto a aspectos económicos como a la aparición de posibles resistencias bacterianas ${ }^{5}$. Estas resistencias se asocian a una mayor morbilidad, mortalidad, demanda sanitaria, coste del tratamiento y deterioro de la calidad del tratamiento de futuros pacientes ${ }^{6-8}$.

La situación del incumplimiento de la terapia antibiótica en España no es bien conocida, pero existen diversos estudios que lo sitúan por encima del $50 \%{ }^{9}$. Otras investigaciones realizadas en varios países europeos mostraron un incumplimiento en nuestro país del $42 \%$, que fue superior al del resto ${ }^{10}$.

Investigaciones realizadas en nuestro país indican que las causas más frecuentes de la no cumplimentación terapéutica son: la curación, la mejoría clínica, los efectos secundarios y la desconfianza en el tratamiento ${ }^{11,12}$. Otros factores implicados son: número de dosis, duración del tratamiento, sabor de la medicación, edad de los cuidadores e incluso la asistencia a centros escolares ${ }^{13}$.

La existencia de un amplio número de métodos de medida de la adherencia terapéutica está justificada por las dificultades de su determinación ${ }^{14}$. Se dispone de métodos directos e indirectos, siendo estos últimos los más utilizados por ser más baratos y más fáciles de usar ${ }^{15}$. Aunque no existe ningún sistema gold-standard para determinar el cumplimiento $^{16}$, la guía NICE afirma que el cumplimiento autoinformado es una herramienta útil para la práctica clínica ${ }^{17}$. Se ha indicado que el test de Morisky-Green puede utilizarse por ser un método poco costoso, informar sobre las posibles causas de incumplimiento y ser fiable cuando el enfermo asegura no cumplir adecuadamente con medicación ${ }^{18}$.

Considerando todo lo expuesto y el escaso número de publicaciones actuales sobre cumplimiento terapéutico en patologías agudas de la infancia, realizamos un estudio con el objetivo de conocer el cumplimiento terapéutico en niños a los que se prescribe tratamiento antibiótico e identificar los factores relacionados con el incumplimiento.

\section{SUJETOS Y MÉTODOS}

El estudio que realizamos fue de tipo descriptivo, observacional y prospectivo. Se llevó a cabo en tres consultas de pediatría de dos centros de salud pertenecientes a dos Gerencias de Atención Integrada de la provincia de Albacete (Albacete y Hellín). Los participantes fueron seleccionados mediante muestreo consecutivo no probabilístico durante el periodo comprendido entre el 28 de febrero y el 15 de abril de 2013. Se incluyeron todos los niños con una edad comprendida entre los 6 meses y los 14 años que acudieron a los centros de salud participantes y que recibieron tratamiento antibiótico por presentar algún tipo de proceso infeccioso agudo en ese momento. Los pacientes fueron evaluados el día que se realizó la prescripción del tratamiento y al cabo de una semana, solicitando previamente el consentimiento a sus padres o tutores para participar en el estudio una vez que se les informó de los objetivos del mismo. Se excluyeron aquellos pacientes que presentaban criterios de hospitalización o cualquier otra circunstancia que les impidiera acudir a la visita de seguimiento. Los padres o acompañantes de los pacientes fueron entrevistados en su centro de salud mediante un cuestionario precodificado, diseñado específicamente para este estudio.

Se midieron las siguientes variables: características sociodemográficas (sexo y edad del niño; nivel de instrucción del padre y la madre; profesión del padre y la madre; estado civil de ambos; tipo de convivencia; número de hermanos del niño; nacionalidad del padre, de la madre y del niño), características del fármaco prescrito (tipo de antibiótico, dosis, posología), patología infecciosa actual por la que se prescribe el antibiótico, problemas de salud crónicos (clasificación CIAP-2), tratamientos habituales, infecciones durante los últimos 6 meses, y frecuentación a la consulta (número de visitas durante el último mes y los últimos 3 meses). Durante 
la visita realizada a los 7 días se midió la adherencia terapéutica mediante el test de Morisky-Green, que consiste en un cuestionario de 4 preguntas dicotómicas referidas al olvido de la medicación y a la adecuación a la pauta, en el que se considera cumplidor al que responde de forma correcta a todas ellas ${ }^{19}$. Otras variables fueron: presencia de efectos adversos manifestados por el paciente o por sus acompañantes, modificación de tratamiento y motivos para no tomar la medicación correctamente si esto ocurrió.

En cuanto al análisis estadístico, se realizó una descripción de las características de los sujetos de la muestra (mediante proporciones, medidas de tendencia central y medidas de dispersión). Para estudiar la asociación entre variables se recurrió a pruebas de independencia para comparar proporciones $\left(x^{2}\right)$ y pruebas de comparación de medias en grupos independientes (t-Student). Por último, mediante análisis multivariante (regresión logística) se comprobó la asociación entre el incumplimiento del tratamiento antibiótico (variable dependiente) y sus posibles factores relacionados, realizando un ajuste estadístico y comprobando la existencia de factores de confusión. En todas las pruebas estadísticas se consideró como nivel de significación $\alpha=0,05$. Los análisis se realizaron mediante el sistema SPSS v 20.0.

\section{RESULTADOS}

Se estudiaron los 117 sujetos a los que se les propuso participar (tasa de aceptación: 100\%). La edad media fue de 4,8 años (DE: 3,3 ) y un $53,3 \%$ fueron niñas. El resto de características de los participantes se muestran en la tabla 1.

Respecto a la frecuentación a la consulta de pediatría, el $48,7 \%$ había acudido en 2 o más ocasiones en el último mes y el $47,9 \%$ en 3 o más ocasiones en los tres meses últimos. En cuanto a los problemas de salud previos, un 23,9\% de los niños había presentado algún tipo de patología no infecciosa, siendo el asma la enfermedad más frecuente, al afectar al $60,7 \%$ de los que presentaban algún problema de salud. Un $24,8 \%$ de los participantes no había presentado ninguna infección previa a la actual en los últimos 6 meses, un 35\% había padecido episodios infecciosos previos en una o dos ocasiones y un $40,2 \%$ los había presentado tres o más veces.

La distribución de las infecciones para las que se prescribió el tratamiento antibiótico fue la siguiente: 47 casos de amigdalitis ( $40,2 \%), 33$ de otitis media
(28,2\%), 22 de infecciones respiratorias $(18,8 \%)$ y 15 de otras infecciones (12,8\%). Los fármacos utilizados con mayor frecuencia fueron amoxicilina con ácido clavulánico $(47,9 \%)$ y amoxicilina (41\%).

El cumplimiento terapéutico mediante el cuestionario de Morisky-Green no fue adecuado en el 55,6\% (IC95\%: 45,1-65,0\%) de los pacientes. Del total de los no cumplidores, el $66,2 \%$ tomó la medicación de forma irregular, el 32,3\% abandonó el tratamiento y el 1,5\% realizó ambas cosas. En los incumplidores, los motivos más frecuentemente manifestados por los padres para la administración incorrecta o abandono del tratamiento se muestran en la tabla 2. Mediante análisis bivariante no se observaron diferencias estadísticamente significativas en el porcentaje de incumplidores respecto al sexo o la edad, ni en los de cada uno de los motivos de incumplimiento respecto a ambas variables.

Refirieron presentar efectos adversos 14 pacientes (12\%), distribuyéndose de la siguiente forma: 10 casos de diarrea, 3 de vómitos y 1 erupción en piel. Se cambió el tipo de antibiótico en 5 casos (4,3\%).

Mediante análisis multivariante (modelo de regresión logística), las variables asociadas al incumplimiento fueron: tener un padre con estudios secundarios o superiores (OR 3,$1 ; p=0,020)$, una madre con estudios primarios o inferiores (OR 3,3; $p=0,010)$ y realizar tres tomas de antibiótico diarias (OR 5,2; $p=0,012$ ), resultando al borde de la significación estadística haber asistido a la consulta del pediatra en 2 o más ocasiones en el último mes (OR 2,2; $p=0,059$ ) (tabla 3).

\section{DISCUSIÓN}

En los resultados obtenidos observamos que más de la mitad de los niños que reciben tratamiento antibiótico para patología agudas no cumplen adecuadamente ese tratamiento. Estos resultados son similares a los de estudios previos que señalan un intervalo de incumplimiento de la terapia antibiótica entre el $40 \%$ y el $70 \%{ }^{20-22}$ o, en un intervalo más amplio, del 25 al $82 \%{ }^{15}$. Tampoco son diferentes de los de otros estudios españoles que señalan un $60 \%$ de incumplimiento del tratamiento en infecciones respiratorias agudas ${ }^{23}$ o que lo sitúan por encima del $50 \%^{9,24}$.

Aunque se ha afirmado que el conocimiento o el nivel de educación no influyen en el cumplimiento terapéutico ${ }^{13}$, en nuestros resultados se ha mostrado que el nivel de estudios de los padres está relacionado con el incumplimiento. Sin embargo, 


\begin{tabular}{|c|c|c|}
\hline Características de los participantes & Número $(n=117)$ & Porcentaje (\%) \\
\hline \multicolumn{3}{|l|}{ Edad } \\
\hline Menor de 2 años & 21 & 17,9 \\
\hline Entre 2 y 4 años & 38 & 32,5 \\
\hline Entre 4 y 6 años & 24 & 20,5 \\
\hline Mayor de 6 años & 34 & 29,1 \\
\hline \multicolumn{3}{|l|}{ Sexo } \\
\hline Niño & 54 & 46,2 \\
\hline Niña & 63 & 53,8 \\
\hline \multicolumn{3}{|l|}{ Nivel de estudios del padre } \\
\hline Primarios o inferiores & 73 & 62,4 \\
\hline Medios o superiores & 44 & 37,6 \\
\hline \multicolumn{3}{|l|}{ Nivel de estudios de la madre } \\
\hline Primarios o inferiores & 55 & 47,0 \\
\hline Medios o superiores & 62 & 53,0 \\
\hline \multicolumn{3}{|l|}{ Estado civil del padre } \\
\hline Casado o unión estable & 100 & 85,5 \\
\hline Soltero, viudo, divorciado y otros & 17 & 14,5 \\
\hline \multicolumn{3}{|l|}{ Estado civil de la madre } \\
\hline Casado o unión estable & 100 & 85,5 \\
\hline Soltero, viudo, divorciado y otros & 17 & 14,5 \\
\hline \multicolumn{3}{|l|}{ Clase social } \\
\hline Clases I-III * & 53 & 45,3 \\
\hline Clases IV-VI ** & 64 & 54,7 \\
\hline \multicolumn{3}{|l|}{ Tipo de convivencia } \\
\hline Con ambos padres & 107 & 91,5 \\
\hline Con uno de los padres & 10 & 8,5 \\
\hline \multicolumn{3}{|l|}{ Nacionalidad del niño } \\
\hline Española & 114 & 97,4 \\
\hline No española & 3 & 2,6 \\
\hline \multicolumn{3}{|l|}{ Nacionalidad del padre } \\
\hline Española & 104 & 88,9 \\
\hline No española & 13 & 11,1 \\
\hline \multicolumn{3}{|l|}{ Nacionalidad de la madre } \\
\hline Española & 104 & 88,9 \\
\hline No española & 13 & 11,1 \\
\hline \multicolumn{3}{|l|}{ Número de hermanos } \\
\hline Ningún hermano & 30 & 25,6 \\
\hline Un hermano & 68 & 58,1 \\
\hline Dos o más hermanos & 16 & 16,3 \\
\hline
\end{tabular}

Tabla 1. Características sociodemográficas de los participantes. * Directivos, funcionarios, profesionales liberales, técnicos superiores, cuadros y mandos intermedios, administrativos, personal de los servicios de protección y seguridad. ** Trabajadores manuales cualificados y trabajadores manuales semicualificados o no cualificados (de la industria, comercio, servicios y sector primario), amas de casa y otros 


\begin{tabular}{lccc}
\hline Motivo de incumplimiento & Total (n=65) & Niños (n=32) & Niñas (n=33) \\
\hline No despertar al niño & $\mathbf{n}(\%)$ & $\mathbf{n}(\%)$ & $\mathbf{n}(\%)$ \\
Presentar mejoría o estar bien & $26(40,0)$ & $14(43,8)$ & $12(36,4)$ \\
Olvido de tomas & $17(26,2)$ & $9(28,1)$ & $8(24,2)$ \\
Presencia de efectos secundarios & $9(13,8)$ & $3(9,4)$ & $6(18,2)$ \\
No ser efectivo el tratamiento & $6(9,2)$ & $3(9,4)$ & $3(9,2)$ \\
NS/NC * & $1(1,5)$ & $1(3,1)$ & $0(0,0)$ \\
\hline
\end{tabular}

Tabla 2. Motivo indicado por los padres para no cumplir adecuadamente con el tratamiento antibiótico en los participantes incumplidores. ${ }^{*}$ NS/NC: No sabe o no contesta

\begin{tabular}{lcccc}
\hline Variables & Coeficiente & OR & IC 95\% & p \\
\hline Padre con estudios secundarios o superiores & 1,13 & 3,11 & $1,19-8,09$ & 0,020 \\
Madre con estudios primarios o inferiores & 1,20 & 3,33 & $1,33-8,32$ & 0,010 \\
Posología de 3 tomas / día & 1,64 & 5,16 & $1,45-18,46$ & 0,012 \\
$\begin{array}{l}\text { Asistencia a consulta de pediatría } \geq 2 \\
\text { ocasiones en el último mes }\end{array}$ & 0,19 & 2,20 & $0,97-5,00$ & 0,059 \\
\hline
\end{tabular}

Tabla 3. Variables asociadas mediante un modelo de regresión logística a incumplimiento del tratamiento antibiótico. IC 95\%: Intervalo de confianza del 95\%. OR: Odds ratio ajustados

se ha asociado con un nivel de estudios secundarios o superiores de los padres, mientras que para las madres esta asociación se ha observado para un nivel de estudios primarios o inferiores. Esta diferencia podría explicarse porque habitualmente son las madres las encargadas de administrar la medicación a los niños. De forma coincidente con lo indicado por otros autores ${ }^{13}$, en nuestro estudio no se ha observado relación entre la adherencia al tratamiento antibiótico y el sexo, edad u otras variables sociodemográficas.

También observamos una asociación estadísticamente significativa entre la prescripción de tres tomas diarias de antibiótico y una inadecuada adherencia al tratamiento. Esta asociación también se ha observado en estudios previos ${ }^{13,15,25}$.

Entre los motivos de incumplimiento, referidos por los padres, se incluyen la mejoría clínica y la pre- sencia de efectos secundarios, que también se han identificado en estudios previos como causas más frecuentes de la no cumplimentación terapéutica ${ }^{11,12}$. Diferentes investigaciones han mostrado que un alto porcentaje de los pacientes piensa que sólo debe continuar el tratamiento con antibióticos mientras tiene síntomas, siendo éste uno de los principales motivos de abandono del tratamiento $26-28$. A pesar de que los efectos adversos referidos fueron de carácter leve y afectaron únicamente a 14 niños, se ha identificado su presencia como uno de los motivos de incumplimiento.

Al analizar los motivos indicados para no cumplir adecuadamente destaca por su frecuencia el de no despertar al niño para la toma, aunque sorprendentemente no se ha observado una relación entre este motivo y la edad del niño. Esto parece contradictorio, pero podría estar relacionado con la distribución por edades de los participantes en nuestro 
caso. Por tanto, es aconsejable realizar nuevos estudios con mayor número de participantes de diferentes edades para comprobar si se mantiene esta ausencia de relación.

Otro de los motivos manifestados en nuestro estudio para no realizar una cumplimentación adecuada es el olvido de tomas. En un metaanálisis previo se observó que más de un tercio de los pacientes se olvidaron de tomar alguna dosis de antibiótico ${ }^{29}$.

Tanto en nuestro estudio como en previos se ha comprobado que mejora la cumplimentación del tratamiento antibiótico cuando se dan menos de 3 tomas al día ${ }^{30}$; por eso parece indicado adecuar el número de tomas para facilitar la administración y mejorar el cumplimiento. Se ha observado que concentraciones de antibióticos superiores al $40-50 \%$ de las dosis habitualmente pautadas son curativas en niños con amigdalitis y otitis medias, permitiendo su administración cada 12 horas $^{31}$. Estas consideraciones deberían tenerse en cuenta al emitir recomendaciones sobre el tratamiento de las enfermedades infecciosas en niños.

Por otra parte, podemos indicar como una limitación de nuestro estudio la utilización de un método para evaluar el incumplimiento que no considera variables cualitativas como las creencias. Además, el test utilizado es un instrumento poco costoso y de gran validez cuando el enfermo asegura no cumplir con la medicación, pero su sensibilidad y valor predictivo negativo son bajos, lo que podría condicionar la interpretación de los resultados cuando al paciente asegura cumplir el tratamiento ${ }^{17,18}$.

Es recomendable la realización de futuros estudios sobre incumplimiento y sus factores asociados que utilicen diferentes tipos de modelos conocidos para detectar a los pacientes con mayor riesgo de presentarlo. Además, son necesarias futuras investigaciones sobre las estrategias dirigidas a mejorar el cumplimiento de este tipo de tratamiento.

En conclusión, más de la mitad de los niños que toma antibióticos en Atención Primaria no cumple correctamente con el tratamiento prescrito. Las variables relacionadas con el incumplimiento deberían considerarse tanto al prescribir estos fármacos como al elaborar las estrategias de mejora del cumplimiento terapéutico en la infancia.

\section{BIBLIOGRAFÍA}

1. Adherence to long term therapies: evidence for action. Geneva: World Health Organization; 2003.
2. Haynes RB. A critical review of the determinats of patient compliance therapeutic regimens. En: Sackett DL, Haynes $\mathrm{RB}$, editors. Compliance with therapeutic regimens. Baltimore: John Hopkins University Press; 1976. p. 24-40.

3. Holmes SJ, Morrow AL, Pickering LK. Child-care practices: effects of social change on the epidemiology of infectious diseases and antibiotic resistance. Epidemiol Rev. 1996; 18: 10-28.

4. Haynes RB, Yao X, Degani A, Kripalani S, Garg A, McDonald HP. Interventions to enhance medication adherence. Cochrane Database Syst Rev. 2005; 4: CD000011.

5. Vrijens B, Urquhart J. Patient adherence to prescribed antimicrobial drug dosing regimens. J Antimicrob Chemother. 2005; 55: 616-27.

6. Smith RD, Coast J. Antimicrobial resistance: a global response. Bulletin of the World Health Organization 2002; 80: 126-33.

7. Lázaro E, Madurga M, de Abajo FJ. Evolución del consumo de antibióticos en España, 1985-2000. Med Clin (Barc). 2002; 118: 561-8.

8. Cars $\mathrm{O}$, Mölstad S, Melander A. Variation in antibiotic use in the European Union. Lancet. 2001; 357: 1851-3.

9. Ramalle E, Bermejo R, Alonso R, Mariño I, Sáenz de Cabezón MI, Villaro C. Cumplimiento del tratamiento antibiótico en niños no hospitalizados. Aten Primaria. 1999; 24: 364-7.

10. Branthwaite AR, Pechere JC. Pan European Survey of Patients. Attitudes to antibiotics and antibiotic use. J Inst Med Res. 1996; 24: 229-38.

11. Alexandre Al. Ensayo clínico de intervención sobre el cumplimiento terapéutico con antibióticos en atención primaria de salud [tesis doctoral]. Universidad Miguel Hernández, San Juan de Alicante; 1999.

12. Segador J. Ensayo clínico de intervención sobre la adhesión al tratamiento antibiótico ambulatorio en faringoamigdalitis aguda [tesis doctoral]. Universidad Miguel Hernández, San Juan de Alicante; 1999.

13. Llor $\mathrm{C}$, Sierra $\mathrm{N}$, Hernández $\mathrm{S}$, Moragas A, Hernández M, Bayona $\mathrm{C}$ et al. The higher the number of daily doses of antibiotic treatment in lower respiratory tract infection the worse the adherence. J Antimicrob Chemother. 2009; 63: 386-9.

14. Basterra M. El cumplimiento terapéutico. Pharm Care Esp. 1999; 1: 97-106.

15. Sclar DA, Tartaglione TA, Fine MJ. Overview of issues related to medical compliance with implications for the outpatient management of infectious diseases. Infect Agents Dis. 1994; 3: 266-73.

16. Vermeire E, Hearnshaw $H$, Van Royen P, Denekens J. Patient adherence to treatment: three decades of research. A comprehensive review. J Clin Pharm Ther. 2001; 26: 33142.

17. National Collaborating Centre for Primary Care: Medicines adherence: Involving patients in decisions about prescribed medicines and supporting adherence London: NICE; 2009. [consultada el 5/12/2013]. Disponible en: http:// www.guidelines.gov/content.aspx?id=14342

18. Gil V, Pineda M, Martínez JL, Belda J, Santos ML, Merino J. Validez de 6 métodos indirectos para valorar el cumplimiento terapéutico en hipertensión arterial. Med Clin (Barc). 1994; 102: 532-6.

19. Morisky DE, Green LW, Levine DM. Concurrent and predictive validity of a self-report measure of medication adherence. Med Care. 1986; 24: 67-74.

20. Sanson-Fisher R, Bowman J, Armstrong S. Factors affecting nonadherence with antibiotics. Diagn Microbiol infect Dis. $1992 ; 151: 103-9$. 
21. Al-Shammari SA, Khoja $\mathrm{T}$, Al-Yamani MJ, Compliance with short-term antibiotic therapy among patients primary health centres in Riyadh, Saudi Arabia. J R Soc Health. 1995; 115 (4): 231-4.

22. Reed BD. Compliance with acute otitis media treatment. J Fam Pract. 1984; 19: 627-32.

23. Reyes $H$, Guiscrafe $H$, Muñoz $O$, Perez Cueva R, Martinez A, Gutierrez G. Antibiotic non-compliance and waste in upper respiratory infections and acute diarrhoea. J Clin Epidemiol. 1997; 50: 1297-304.

24. Martínez-Mir I, Palop V. El problema del incumplimiento terapéutico en diferentes áreas. En: Sacristán JA, García FM, Martínez Mir I, Palop V, Amado E, editores. Cumplimiento Terapéutico. Madrid: Biblioteca Lilly; 2001. p. 73-86.

25. Silvestre $\mathrm{C}$, Ramalle E, Arnáez R, Flor A, Gascía J, Ramil H et al. Estudio multicéntrico sobre adhesión al tratamiento antibiótico en población infantil en Atención Primaria. Aten Primaria. 2001; 27: 554-8.

26. Pichichero ME, Casey JR, Mayes $T$, Francis AB, Marsocci $\mathrm{SM}$, Murphy AM et al. Penicillin failure in streptococcal ton- sillopharyngitis: causes and remedies. Pediatr Infect Dis J. 2000; 19: 917-23.

27. Gil VF, Payá MA, Asensio MA, Torres MT, Pastor R, Merino J. Incumplimiento del tratamiento con antibióticos en infecciones agudas no graves. Med Clin (Barc). 1999; 112: 731-3.

28. Clemente E, Millaina G, Moreno E, Vacas AL. Sobre la "cultura antibiótica" de la población. Aten Primaria. 2000; 26: 136.

29. Kardas P, Devine S, Golembesky A, Roberts C. A systematic review and meta-analysis of misuse of antibiotic therapies in the community. Int J Antimicrob Agents. 2005; 26: 106-13.

30. Kardas P. Comparison of patient compliance with oncedaily and twice-daily antibiotic regimens in respiratory tract infections: results of a randomised trial. J Antimicrob Chemother. 2007; 59: 531-6.

31. Álvez F. Uso racional de antibióticos en las infecciones más comunes de los niños. An Pediatr Contin. 2010; 8: 221-30. 